DOE/ER/40427-08-N94

\title{
MESONS AND THE STRUCTURE OF NUCLEONS*
}

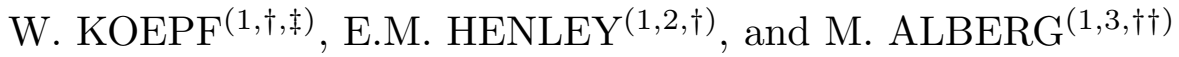 \\ (1) Department of Physics, FM-15, University of Washington \\ Seattle, Washington 98195 USA \\ ${ }^{(2)}$ Institute for Nuclear Theory, HN-12, University of Washington \\ Seattle, Washington 98195 USA \\ (3) Department of Physics, Seattle University, Seattle, WA 98122 USA
}

\begin{abstract}
The role of mesons, particularly the pion, in the structure of nucleons is reviewed and investigated. Since quark-antiquark pairs are likely to "transform" into mesons at large distances, mesons are expected to contribute to nucleon structure. Their effects on the Gottfried sum rule, on the strangeness content of the nucleon, and on the spin of the nucleon are discussed.
\end{abstract}

\section{Introduction}

Ever since the postulation of mesons by Yukawa in 1934, and the discovery of the pion in 1947, it has been clear that mesons play a crucial role in the structure of the nucleon and in hadronic forces. The long range part of the nucleon-nucleon interaction is clearly dominated by meson exchanges. The puzzle of the shortdistance behavior of nuclear forces has been replaced by our lack of knowledge of the transition from quarks and gluons to nucleons and mesons. Although it is now accepted that QCD is the basis of hadronic forces, the translation of that knowledge to calculations of hadronic properties is beset by difficulties. Thus, models of the

* It is an honor for us to dedicate this article to the memory of Bob Marshak, who was a direct inspiration to one of us and a mentor to many young physicists.

${ }^{\dagger}$ Supported in part by the Department of Energy, Grant No. DE-FG06-88ER40427. $\ddagger$ Supported in part by the Deutsche Forschungsgemeinschaft and by the FeodorLynen program of the Alexander von Humboldt-Stiftung.

${ }^{\dagger}$ Supported in part by the National Science Foundation, Grant No. PHY-9223618. 
structure of nucleons abound. Many of these models make use of constituent quarks, which represent quarks "dressed" with gluons and quark-antiquark pairs. Since mesons are composed of these pairs, it is natural to believe that at large distances $(\approx 1 \mathrm{fm})$ the quark-antiquark pairs become mesons. Direct evidence for the role of the pion in nucleon structure comes, for instance, from the charge form factor of the neutron, $G_{E}^{n}$, at small momentum transfers. ${ }^{1}$ The negative charge of the pion $\left(n \rightarrow p \pi^{-}\right)$is responsible for the long range part of the charge distribution and accounts for the experimentally observed negative charge radius of the neutron.

Further evidence for the role of mesons in nucleon structure includes a) the success of models in which they play an explicit role, such as the cloudy bag model; ${ }^{1}$ and $b$ ) the experimental value for the Gottfried sum rule. ${ }^{2}$ In addition, the EMCinspired "spin crisis" led to the proposition that strange quarks contribute nonnegligibly to the spin of the nucleon and that only a small fraction of the nucleon's spin is due to the valence quarks. ${ }^{3,4}$ These conjectures brought about suggestions for further experiments to measure the strangeness "content" of the nucleon ${ }^{5-7}$ and the corresponding unknown form factors; ${ }^{7}$ they also led to calculations of the meson contribution to the strangeness of the nucleon, ${ }^{8}$ and to more recent measurements in deep inelastic scattering on ${ }^{3} \mathrm{He}$ and deuterons. ${ }^{9}$

In this paper we intend to review and expand on some of this work.

\section{Mesons and the Gottfried Sum Rule}

The Gottfried sum rule relates to the $F_{2}$ structure functions of the proton and neutron ${ }^{10}$,

$$
S_{G}=\int_{0}^{1} d x \frac{\left[F_{2}^{p}(x)-F_{2}^{n}(x)\right]}{x},
$$

where $F_{2}$ is determined from deep inelastic charged ( $\mu$ or $e$ ) lepton-nucleon scattering. In the quark-parton model, $S_{G}$ can be written as

$$
S_{G}=\sum_{i}\left(\frac{e_{i}}{e}\right)^{2} \int_{0}^{1}\left[q_{i}^{p}(x)+\bar{q}_{i}^{p}(x)-q_{i}^{n}(x)-\bar{q}_{i}^{n}(x)\right] d x
$$

where $e_{i} / e$ is the charge of quark $i$ in units of the elementary charge. Since charge symmetry has been shown to be valid for hadrons to $\lesssim 1 / 2 \%$, we accept it here; it follows that $d^{n}=u^{p} \equiv u, \bar{d}^{n}=\bar{u}^{p} \equiv \bar{u}, u^{n}=d^{p} \equiv d$, and $\bar{u}^{n}=\bar{d}^{p} \equiv \bar{d}$. However charge symmetry does not imply flavor independence of the sea, i.e., $\bar{u}=\bar{d}$. With these simplifications, Eq. (2) becomes

$$
S_{G}=\frac{1}{3}+\frac{2}{3} \int_{0}^{1}[\bar{u}(x)-\bar{d}(x)] d x=\frac{1}{3}(1-\Delta \bar{q})
$$


where the last integral is the sea quark contribution. The experimental result of the NMC group, ${ }^{2} S_{G} \approx 0.240 \pm 0.016$ at $Q^{2}=5 \mathrm{GeV}^{2}$, leads to the conclusion that there are more $\bar{d}$ quarks than $\bar{u}$ quarks in the sea of the proton $(\Delta \bar{q}=0.280 \pm 0.048)$, unless charge symmetry fails. This result appears at first sight to be a puzzle, since gluons would be expected to generate an equal number of $u \bar{u}$ and $d \bar{d}$ pairs if quark mass difference effects are neglected. However, as first pointed out by Field and Feynman, ${ }^{11}$ the Pauli exclusion principle, alone, would give an excess of $d \bar{d}$ pairs over $u \bar{u}$ pairs in the proton sea since there are already two valence $u$ quarks and only one $d$ quark in the proton. However, it is doubtful that this effect would lead to as large a reduction of $S_{G}$ as observed. On the other hand, the pion cloud contribution also affects $S_{G}$. Although the $\pi^{0}$ has an equal number of $\bar{u}$ and $\bar{d}$ quarks, the proton has an excess of $\pi^{+}(u \bar{d})$ over $\pi^{-}(d \bar{u})$ and thus more $\bar{d}$ quarks than $\bar{u}$ quarks. ${ }^{12}$ Of course, when the $\pi^{+}$is "in the air", the valence quark distribution of the proton is altered; however, for small $x$, the distinction between valence and sea quarks is difficult to maintain. On the constituent quark level, the above process is $u \rightarrow d+(u \bar{d})$. Since there are more $u$ quarks than $d$ quarks $(d \rightarrow u+d \bar{u})$, the same asymmetry holds at the constituent quark level.

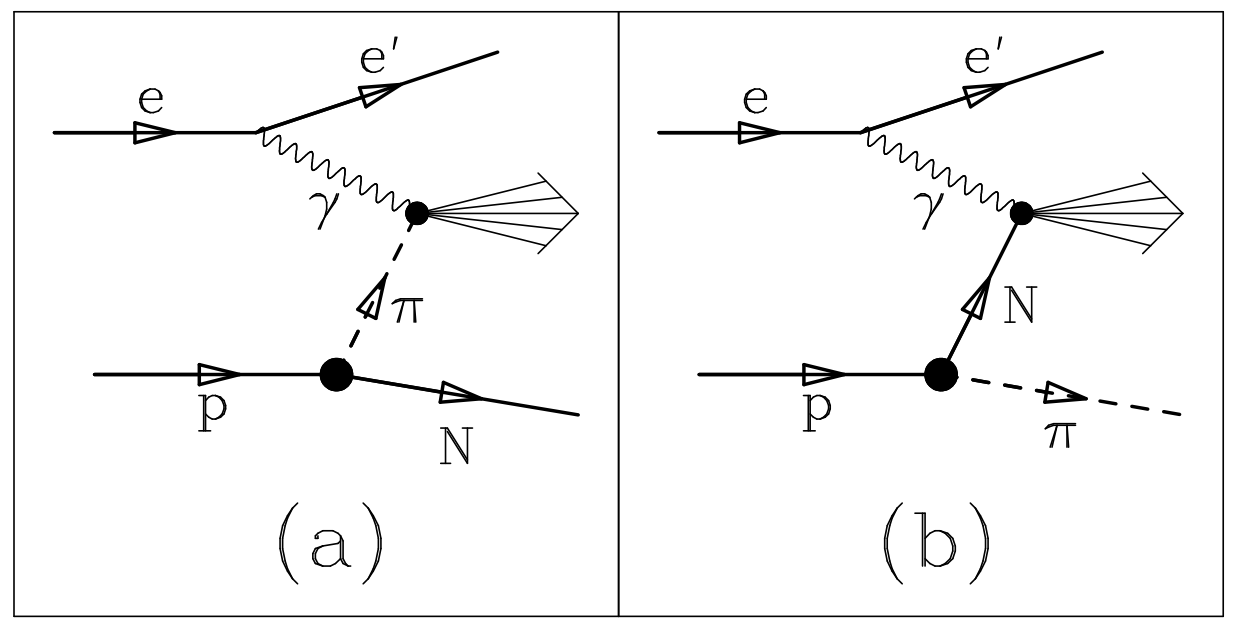

Figure 1. The pion cloud contribution to the proton structure function in deep inelastic scattering from the $p \rightarrow N+\pi$ process.

The meson cloud contribution to the structure function was considered by Sullivan ${ }^{13}$ over 20 years ago and has received considerable revived interest in the past few years. ${ }^{12,14}$ The pionic contribution, Figs. 1 and 2, to the proton structure function in deep inelastic scattering can be written as a convolution ${ }^{12,14}$ of the pion structure function, $F_{2}^{\pi}$, and its momentum distribution, $f_{\pi}$, in the infinite 


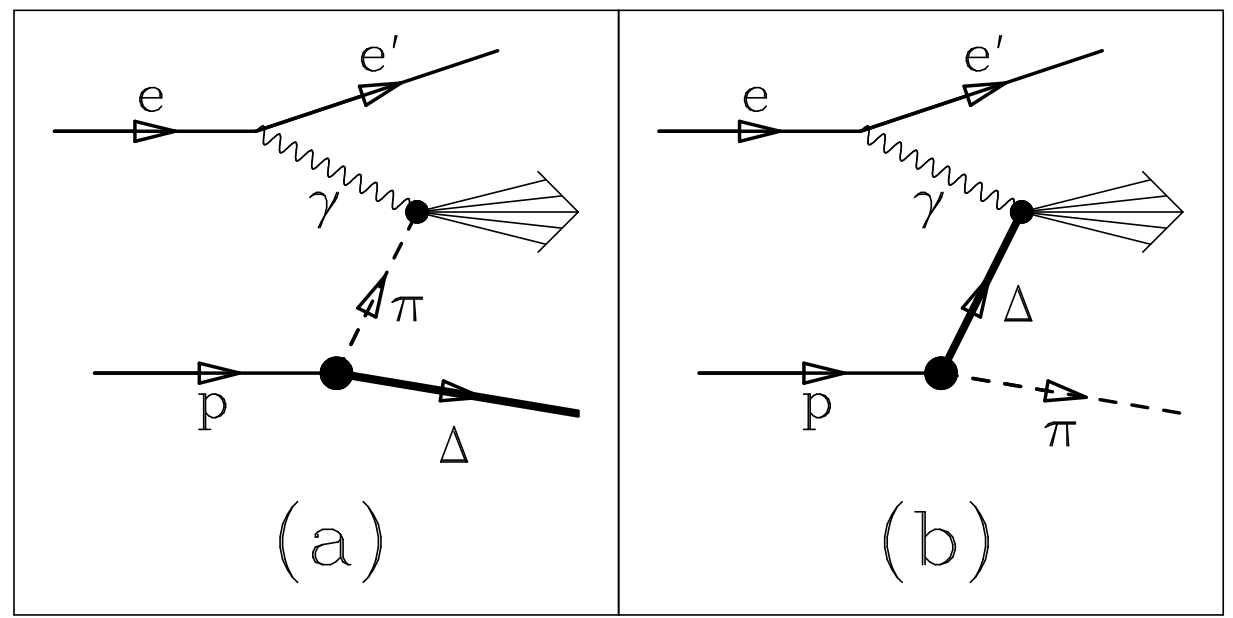

Figure 2. Same as Fig. 1, but from the $p \rightarrow \Delta+\pi$ process.

momentum frame. We find,

$$
\begin{aligned}
\Delta F_{2}^{p}(x) & =\int_{x}^{1} d y f_{\pi}(y) F_{2}^{\pi}\left(\frac{x}{y}, Q^{2}\right)+\int_{0}^{1-x} d y f_{\pi}(y) F_{2}^{N}\left(\frac{x}{1-y}, Q^{2}\right), \\
f_{\pi}(y) & =\frac{3 g_{\pi N}^{2}}{(4 \pi)^{2}} \int_{\frac{M^{2} y}{1-y}}^{\infty} \frac{t}{\left(t+m_{\pi}^{2}\right)} F_{\pi N}^{2}(t) d t
\end{aligned}
$$

where $g_{\pi N}$ is the pion-nucleon coupling constant, $M$ the nucleon mass, and $F_{\pi N}$ is the pion-nucleon form factor. The first term in Eq. (4) comes from Fig. 1a and the second one from Fig. 1b, where the photon couples to the recoil nucleon. In our approach, when the difference between a proton and neutron is taken, the symmetry of a photon striking the $\pi^{+}$in the proton and the $\pi^{-}$in the neutron cancels this contribution to the Gottfried sum rule and leaves only the contribution of the second term in Eq. (4), shown in Fig. 1 b.

In addition to the contribution of Fig. 1 to the structure function, pion emission from a nucleon can lead to excited states. The most important of these is likely to be that of lowest energy, the $\Delta$, where $p \rightarrow \Delta^{++} \pi^{-}, \Delta^{+} \pi^{0}, \Delta^{0} \pi^{+}$and $n \rightarrow$ $\Delta^{-} \pi^{+}, \Delta^{0} \pi^{0}$. Here, the contribution of the pion cloud, shown in Figs. 2a and $2 \mathrm{~b}$, tends to increase $S_{G}$ above $1 / 3$. The contribution to $\Delta F_{2}$ is similar to Eqs. (4) and (5) with $N$ replaced by $\Delta$. The structure function of the $\Delta$ is less well known than that of the nucleon and the pion and thus there is more uncertainty in calculating this contribution. However, it is expected to be smaller due to the mass difference of the nucleon and delta states which increases the minimum value of $t$ in Eq. (5) to $\left[M_{\Delta}^{2}-(1-y) M^{2}\right] y /(1-y)$. We neglect higher mass resonances and other mesons. 
If we include the pion contribution, the dressed nucleon state function $|N\rangle$ is

$$
\left.\left.\left.|N\rangle=\sqrt{\mathcal{N}}\{\mid N)+\sqrt{n_{\pi}} \mid N \pi\right)+\sqrt{\Delta_{\pi}} \mid \Delta \pi\right)\right\}
$$

where $\mid N)$ is a bare nucleon. The structure function $F_{2}$ is then renormalized by the factor $\mathcal{N}=\left(1+n_{\pi}+\Delta_{\pi}\right)^{-1}$, with $n_{\pi}=\int_{0}^{1} d y f_{\pi}(y) d y$ and similarly for $\Delta_{\pi}$. The technical details are described in Refs. 12-14. With these approximations, the Gottfried sum rule becomes

$$
S_{G}=\mathcal{N}\left(\frac{1}{3}-\frac{n_{\pi}}{9}+\frac{5 \Delta \pi}{9}\right) .
$$

The numerical value of $S_{G}$ depends on the form factor $F_{\pi N}$; the higher the effective momentum cutoff, the larger the reduction of $S_{G}$ from $1 / 3$; the experimental value of 0.24 can be reached for a cutoff of $\approx 1.5 \mathrm{GeV}$.

In our opinion, the two mechanisms, the Pauli principle and the pion cloud, offer natural explanations for the measured reduction of the Gottfried sum rule from the anticipated value of $1 / 3$.

Recent experiments have attempted to measure the pion contribution to the excess of $\bar{d}$ (over $\bar{u}$ ) quarks in a Drell-Yan reaction. ${ }^{15}$ Although no asymmetry from the pions was observed, this conclusion may not be inconsistent with the results of the NMC group and our interpretation thereof. ${ }^{16}$

\section{Mesons and Strangeness in the Nucleon}

There has been considerable interest in the strangeness matrix elements in the nucleon, sparked, on the one hand, by a determination of the strange quark scalar density, $\langle N|\bar{s} s| N\rangle$, from the pion-nucleon sigma term. Although the interpretation of the experimental results has been the source of much debate, ${ }^{17}$ it points at a sizeable value of $\langle N|\bar{s} s| N\rangle$, which, in turn, may be the reason behind violations of the OZI rule observed in the $\bar{p} p \rightarrow \Phi+X$ reaction. ${ }^{18}$ Knowledge of the matrix element $\langle N|\bar{s} s| N\rangle$ would also be of importance in determining the critical density for kaon condensation in dense nuclear matter and hence for the occurrence of enhanced strange particle production in heavy ion collisions. ${ }^{19}$

In addition, there exists experimental evidence for a non-vanishing strange quark axial vector matrix element, $\left\langle N\left|\bar{s} \gamma_{\mu} \gamma_{5} s\right| N\right\rangle$, through the BNL low-energy elastic neutrino-proton cross sections ${ }^{20}$ as well as from the EMC measurements ${ }^{3}$ of the spin dependent structure function of the proton in deep inelastic scattering of polarized muons on polarized hydrogen. The latter determination of the strange 
axial vector form factor relies on $S U(3)$ symmetry arguments, and it has been reexamined through measurements carried out recently by the $\mathrm{SMC}^{21}$ and the E142 ${ }^{22}$ groups on deep inelastic scattering of polarized leptons on a polarized deuteron and ${ }^{3} \mathrm{He}$ target, respectively. See Refs. 9 and 23 for clear discussions on this topic.

Furthermore, the goal of the SAMPLE experiment presently underway at MITBates $^{24}$ as well as of three other experiments employing parity violating electron scattering on either protons or ${ }^{4} \mathrm{He}$ planned for $\mathrm{CEBAF}^{25}$ is to constrain the strange quark vector matrix element, $\left\langle N\left|\bar{s} \gamma_{\mu} s\right| N\right\rangle$. Also, a new determination of the strange quark axial vector form factor at a significantly lower momentum transfer than in the original BNL measurements is expected from the LSND experiment at LAMPF, ${ }^{26}$ and there are further suggestions for even more exotic measurements of the various strange quark matrix elements in the nucleon. ${ }^{5}$

From a theoretical standpoint, the existence of significant strange quark matrix elements in the nucleon is rather surprising, especially in the light of the success with which naive constituent quark models - which inherently disregard the existence of any "strangeness" in the nucleon - account for most low-energy properties of the baryonic octet. In a recent article, Karl pointed out that there is actually no contradiction between the baryon magnetic moments and the existence of nonvanishing strangeness matrix elements in the nucleon, even in a constituent quark picture, if one is willing to accept that the constituent quarks themselves have a non-trivial structure. ${ }^{27}$ Steininger and Weise showed that such a picture arises quite naturally in a chiral quark model, ${ }^{28}$ and Kaplan and Manohar pointed out that a different multiplicative renormalization in different flavor $S U(3)$ representations as generated by the $U_{A}(1)$ axial anomaly can lead to a non-trivial flavor structure of the constituent quarks, and hence to strange quark matrix elements in non-strange hadrons. ${ }^{4}$

Aside from those more qualitative than quantitative investigations, there exists, however, only a handful of theoretical calculations that present an estimate of the strangeness matrix elements of the nucleon. There is Jaffe's pole analysis ${ }^{29}$ where the strangeness radius and the strange quark magnetic moment were obtained using three-pole dispersion theory fits to the nucleon's isoscalar form factor, together with a standard treatment of the $\Phi-\omega$ mixing and some mild assumptions on the asymptotic behavior of the nucleon form factors. Similar ideas were utilized in a calculation by Cohen et al., ${ }^{30}$ where the authors consider the strangeness vector current in the nucleon as generated through the $\Phi-\omega$ mixing in a model based on vector meson dominance (VMD). In addition, there are both Skyrme ${ }^{31}$ as well as 
kaon loop model ${ }^{8,32,33}$ estimates of the various strange quark matrix elements in the nucleon.

In the following, we will concentrate on the amount of intrinsic strangeness which is generated in the nucleon through its virtual kaon cloud, i.e., by means of the dissociation of a nucleon into a strange baryon plus a kaon, as e.g., by the process

$$
p \rightarrow \Lambda+K^{+} \rightarrow p
$$

In such a picture, one should not at all be surprised that strange quark matrix elements are non-vanishing, since there exist substantial $\hat{J}_{s}=\bar{s} \Gamma s$ couplings (with $\Gamma=1, \gamma_{\mu}$ or $\left.\gamma_{\mu} \gamma_{5}\right)$ to the kaon as well as to the strange hyperons of the baryonic octet and decuplet. In Refs. 8, 32, and 33, the nucleon's strange quark matrix elements which arise from its virtual kaon cloud were studied in one-loop calculations, and each time quite different prescriptions for the evaluation of the relevant loop integrals were employed.

In the first part of Ref. 8, a single meson-loop model was studied in covariant perturbation theory, and pseudoscalar point couplings between free nucleon and meson fields were used. The meson cloud contribution to both the usual electromagnetic as well as to the strange magnetic moment was calculated, since the corresponding form factor, $F_{2}$, is explicitly finite even without any cutoff. The model, which treats the bare hadrons as pointlike structureless particles, leads to a significant strangeness content $\left(F_{2}^{s}(0) \approx-1.0 \mu_{N}\right)$ but it gives very unsatisfactory results for the magnetic moments, especially in the isoscalar channel. We find $\kappa^{I S} \equiv \kappa^{p}+\kappa^{n}=-2.4$, which is an order of magnitude larger than the experimental value of -0.12 .

The kaon loops in this model contribute at a sizeable level, because the relevant integrals are dominated by the baryonic masses; yet the model fails to provide a quantitative description of the standard electromagnetic properties of the nucleon. This arises, in part, because the model does not properly take into account the underlying nucleon structure and its spatial extension. When the quark sub-structure is considered, it is the size of the proton, rather than its mass, that determines the effective momentum cutoff.

In the second part of Ref. 8, the authors thus study models which include such a cutoff - as set by confinement - and which have been shown to give a very good description of the static properties of the nucleon, namely an $S U(3)$ extended cloudy bag model $(\mathrm{CBM})^{34}$ as well as a chiral non-relativistic constituent quark model (CNRQM). ${ }^{35}$ The only free parameter in the model - the MIT bag radius for 
the CBM and the oscillator length for the chiral non-relativistic constituent quark model - was adjusted to give an optimal description of the standard electromagnetic observables, i.e. the charge radii and magnetic moments of both the neutron and proton. Predictions were then made for the strange quark contributions to the weak magnetic form factor, $F_{2}^{s}(0)$, the strangeness radius, $\left\langle r^{2}\right\rangle_{s}$, and the strangeness axial vector matrix-element, $g_{A}^{s}$. The corresponding results, including some "seagull" gauge invariance corrections which were not considered in the original work in Ref. 8 , will be presented at the end of this section for a MIT bag radius of $R \approx 1.1 \mathrm{fm}$ adjusted to fit the electromagnetic observables and which, in turn, corresponds to an effective three-momentum cutoff of approximately $0.2 \mathrm{GeV}$. For further details see Ref. 8 and the references therein.

In the meson-loop calculation of Musolf and Burkardt, ${ }^{32}$ on the other hand, no attempt was made to reproduce the electromagnetic form factors. Rather, the authors employ an effective meson-nucleon vertex characterized by a monopole form factor

$$
F\left(k^{2}\right)=\frac{m^{2}-\Lambda^{2}}{k^{2}-\Lambda^{2}},
$$

where the ultraviolet cutoff parameter $\Lambda$ is obtained from nucleon-nucleon and nucleon-hyperon scattering, and is typically in the range of 1 to $2 \mathrm{GeV}$. In a similar evaluation of the kaon loop contribution, Holstein ${ }^{8}$ equates the UV cutoff parameter with the nucleon mass, and he finds a moderate value for the strange axial vector form factor of $g_{A}^{s} \approx-0.1$.

In Table 1, we summarize the various estimates for the strange quark form factors of the nucleon deduced both from experiments as well as from some of the theoretical model calculations discussed above. Recently, Phatak and Sahu ${ }^{33}$ evaluated the nucleonic strangeness content in a $S U(3)$ chiral color dielectric model (CCDM), which is in spirit very similar to the extended cloudy bag model which was studied in Ref. 8. Note, however, that both in Ref. 33 as well as in the original work in Ref. 8, the Ward-Takahashi identity was not satisfied, and that the corresponding "seagull" corrections have an appreciable effect on the matrix elements under consideration. ${ }^{8,32}$ For further details on the issue of gauge invariance in chiral hybrid quark models see Refs. 8 and 32, and references therein.

As expected, the "virtual kaon loop" estimates are rather sensitive to the momentum cutoff used in the effective meson-nucleon vertices, and they diminish rapidly with decreasing cutoff parameter $\Lambda$. Except for the calculation employing point couplings for these vertices, which, in turn, leads to quite unsatisfactory 
Table 1. Estimates for the strange quark vector and axial vector matrix elements in the nucleon, deduced both from experiment (the first two rows) as well as from different hadronic models. The last four rows correspond to the "virtual kaon loop" calculations. Results from Ref. 32 were obtained with a cutoff of $\Lambda \approx 1.3 \mathrm{GeV}$.

\begin{tabular}{l|cc|c}
\hline & \multicolumn{2}{|c|}{$\left\langle N\left|\bar{s} \gamma_{\mu} s\right| N\right\rangle$} & $\left\langle N\left|\bar{s} \gamma_{\mu} \gamma_{5} s\right| N\right\rangle$ \\
& $F_{2}^{s}(0)\left[\mu_{N}\right]$ & $\left\langle r^{2}\right\rangle_{s}\left[\mathrm{fm}^{2}\right]$ & $g_{A}^{s}$ \\
& & & $-0.15 \pm 0.09$ \\
BNL $^{20}$ & & & $-0.19 \pm 0.06$ \\
EMC $^{3}$ & & & \\
Poles $^{29}$ & $-0.31 \pm 0.09$ & $0.14 \pm 0.07$ & \\
VMD $^{30}$ & $-0.33 \ldots-0.13$ & $-0.21 \ldots-0.11$ & -0.10 \\
Skyrme $^{31}$ & -1.0 & & -0.04 \\
Point Couplings $^{8}$ & -0.3 & -0.03 & -0.004 \\
Monopole Cutoff $^{32}$ & -0.09 & -0.006 & -0.009 \\
Cloudy Bag $^{8}$ & -0.03 & -0.004 & \\
CCDM $^{33}$ & & & \\
\hline
\end{tabular}

predictions for the standard electromagnetic observables, the strange quark matrix elements that were obtained in the loop analyses are significantly smaller than the ones found either in Jaffe's pole analysis ${ }^{29}$ or in the $S U(3)$ Skyrme model calculations. $^{31}$

This is not at all surprising, since in most models discussed here the mesonic loops yield only small corrections to hadronic models that themselves were designed to already fit the bulk of the low-energy properties of the baryonic octet. Due to the relatively high mass of the kaon, when compared e.g., with a natural energy scale set by the size of the nucleon or the mass of the pion, its contribution - and hence the amount of strangeness in the nucleon generated by means of virtual kaon loops - will be rather small.

We are well aware that the loop calculations discussed here can only yield a crude estimate for the strange quark matrix elements under consideration. Not only do they attempt to use a perturbative expansion for intrinsically strongly 
coupled physics, but they also neglect the contribution from the $U_{A}(1)$ axial anomaly and still employ the traditional picture of a nucleon built from three non-strange valence quarks, and thus discard any intrinsic strangeness in the constituent quarks themselves, as suggested e.g., by $\mathrm{Karl}^{27}$ or by Kaplan and Manohar. ${ }^{4}$ However, we believe that they point to evidence for significant meson contributions to the nucleon structure.

\section{Mesons and the Spin of the Nucleon}

In the previous two sections we have discussed how the meson cloud contributes to a reduction of $S_{G}$ in the Gottfried sum rule and to the existence of non-zero strange quark matrix elements in the nucleon. The cloud also affects the spin of the nucleon, since the dressed nucleon state (Eq. (6)) includes terms in which mesons carry orbital angular momentum and thereby depolarize the nucleon. Mesons play an important role in understanding the alleged "spin crisis" created by the EMC measurements ${ }^{3}$ and further studied in the $\mathrm{SMC}^{21}$ and $\mathrm{E} 142^{22}$ experiments. In the following, we review the experimental determination of the nucleon spin and the theoretical interpretation of these results.

In the EMC experiment, measurements of deep inelastic scattering of polarized muons from polarized hydrogen targets were used to determine the asymmetry

$$
A=\frac{\sigma^{\uparrow}-\sigma^{\downarrow}}{\sigma^{\uparrow}+\sigma^{\downarrow}},
$$

in which $\sigma^{\uparrow(\downarrow)}$ is the cross section for muons polarized parallel (antiparallel) to the spin of the proton. From the asymmetry $A\left(x, Q^{2}\right)$ and the unpolarized structure function $F_{1}\left(x, Q^{2}\right)$ one can determine the polarized structure function $g_{1}\left(x, Q^{2}\right)$, where $x$ is the Bjorken scaling variable, through

$$
g_{1}\left(x, Q^{2}\right)=F_{1}\left(x, Q^{2}\right) A\left(x, Q^{2}\right)
$$

In the limit of asymptotic momentum transfer, the polarized structure function $g_{1}(x)$ is determined by the distribution functions $q_{i}^{\uparrow(\downarrow)}(x)$ of a quark $i$ with spin parallel (antiparallel) to that of the proton,

$$
g_{1}(x)=\frac{1}{2} \sum_{i} e_{i}^{2}\left[q_{i}^{\uparrow}(x)-q_{i}^{\downarrow}(x)\right] .
$$

The spin of the proton can be decomposed into 3 terms,

$$
\frac{1}{2}=\frac{1}{2} \sum_{i} \Delta q_{i}+\Delta G+\left\langle L_{z}\right\rangle
$$


which correspond to the quark, gluon and orbital angular momentum contributions, respectively. The quark contributions

$$
\Delta q_{i}=\int_{0}^{1} d x\left(q_{i}^{\uparrow}+\bar{q}_{i}^{\uparrow}-q_{i}^{\downarrow}-\bar{q}_{i}^{\downarrow}\right)+O\left(\alpha_{s} / \pi\right)
$$

are related to the first moments $\Gamma_{1}$ of the experimentally determined polarized structure functions. In the asymptotic limit, we have e.g.,

$$
\Gamma_{1}^{p}=\int_{0}^{1} g_{1}^{p}(x) d x=\frac{1}{2}\left(\frac{4}{9} \Delta u+\frac{1}{9} \Delta d+\frac{1}{9} \Delta s\right) .
$$

These first moments can be used, together with weak coupling constants extracted from neutron and hyperon decays, to determine the individual contributions $\Delta u, \Delta d$, and $\Delta s$.

The EMC experiment found

$$
\Gamma_{1}^{p}=0.126 \pm 0.011 \pm 0.014
$$

whereas the Ellis-Jaffe sum rule, ${ }^{36}$ which assumes $\Delta s=0$, gives

$$
\Gamma_{1}^{p}=0.175 \pm 0.007
$$

This was the origin of the "spin crisis". The experimental result indicated that almost none of the proton's spin was carried by its valence quarks, and that $\Delta s$ was significantly non-zero. Intense theoretical and experimental activity was stimulated. In particular, a measurement of the neutron structure function $g_{1}^{n}$ was sought, since the most fundamental sum rule, derived by Bjorken ${ }^{37}$, relates the difference of the first moments of the proton and neutron structure functions to the isovector axial vector coupling constant,

$$
\Gamma_{1}^{p}-\Gamma_{1}^{n}=\int_{0}^{1}\left[g_{1}^{p}\left(x, Q^{2}\right)-g_{1}^{n}\left(x, Q^{2}\right)\right] d x=\frac{g_{A}}{6}\left(1-\frac{\alpha_{s}\left(Q^{2}\right)}{\pi}+O\left(\alpha_{s}^{2}\right)\right)
$$

The SMC experiment (deuteron target) and E142 experiment ( ${ }^{3} \mathrm{He}$ target) were designed to provide data on the neutron structure functions. Their results appeared to contradict one another, with SMC agreeing qualitatively with the EMC measurements, but E142 finding that the quarks carried about 1/2 of the nucleon spin and $\Delta s$ consistent with zero. However, the EMC, SMC, and E142 experiments were carried out at different values of $Q^{2}$, and since the $\Gamma_{1}^{p, n}$ are $Q^{2}$ dependent, care 
must be taken to evolve data to the same value of $Q^{2}$ before the first moments are taken. Close and Roberts, ${ }^{23}$ Ellis and Karliner ${ }^{38}$ and $\mathrm{SMC}^{39}$ have reanalyzed all the existing data. They take into account improved determinations of $F_{1}\left(x, Q^{2}\right)$ from the NMC group, errors introduced by extrapolations to low and high values of $x$, and they include leading order QCD corrections. They conclude that the combined experimental data is consistent with the Bjorken sum rule. Their analyses also show that the fraction of the nucleon spin carried by quarks is $Q^{2}$ dependent, decreasing with increasing $Q^{2}$, and that the strange quark contribution is significantly non-zero.

If the quark contribution to the nucleon's spin,

$$
\Delta \Sigma \equiv \sum_{i} \Delta q_{i}
$$

in Eq. (13) is small, then the other terms, $\Delta G$ and $\left\langle L_{z}\right\rangle$, must compensate. Contributions to $\left\langle L_{z}\right\rangle$ are generated by relativistic effects and the meson cloud. Confinement of the quarks in the nucleon leads to non-zero lower components of the spinor wave functions, which carry angular momentum even for quarks in a $s_{1 / 2}$ state, and the $\bar{q} q$ pairs in the meson cloud also carry orbital angular momentum and thus depolarize the nucleon. These effects have been evaluated in the model discussed in Ref. 8.

In the non-relativistic quark model, $\Delta \Sigma$, the fraction of the nucleon's spin carried by the valence quarks, is equal to one. In the MIT bag model, $\Delta \Sigma=0.65$ through the aforementioned relativistic effects. These values are changed in the one-meson-loop calculations of Ref. 8 through couplings to the baryonic octet and decuplet and simultaneous emission of a virtual pion or kaon. Coupling to the octet reduces the axial current of the nucleon, whereas coupling to the decuplet enhances it. The leading order contribution is that of the octet, so the overall effect is a reduction of $\Delta \Sigma$ that depends on the spatial extent of the nucleon, characterized e.g., by the bag radius or oscillator length. In Fig. 3, we show predictions for $\Delta \Sigma$ for the extended cloudy bag model of Ref. 34 and for the chiral non-relativistic constituent quark model of Ref. 35. As expected, $\Delta \Sigma$ is decreased by the oneloop corrections, and the size of the effect decreases with increasing nucleon size. The reduction is about $15 \%$ for nucleon size parameters (MIT bag radius $R \approx 1.1$ fm or oscillator length $r \approx 0.8 \mathrm{fm}$ ) which give a good description of the standard electromagnetic observables. The dashed lines show the valence quarks' contribution to the nucleon spin without the depolarization effect of the mesonic cloud. In this figure, we also depict the average number of "mesons in the air",

$$
\langle n\rangle=n_{\pi}+n_{K}+\Delta_{\pi}+\Delta_{K}
$$



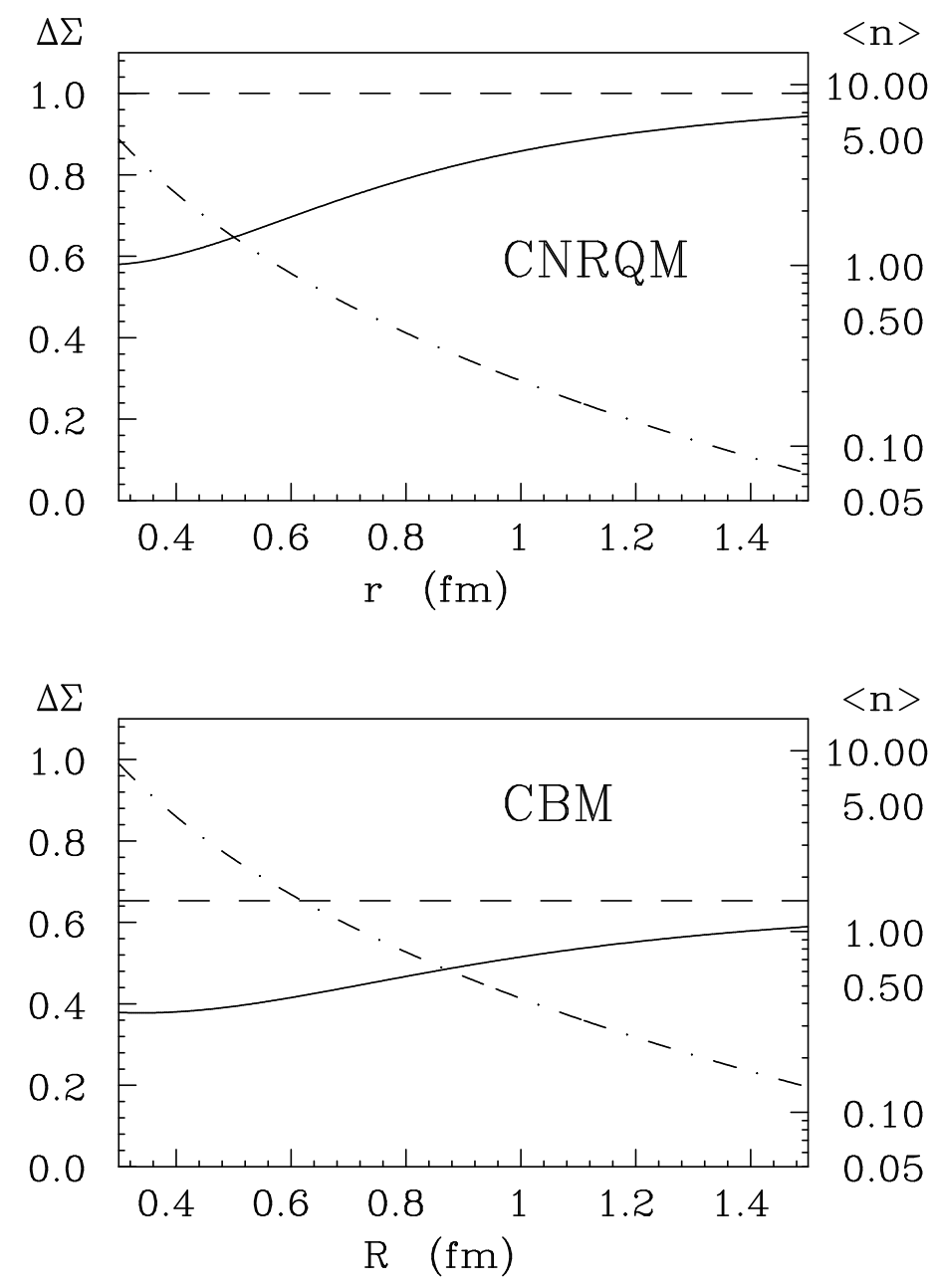

Figure 3. The share of the nucleon's spin carried by its valence quarks (solid line, left scale) and the average number of "mesons in the air" (dotdashed line, right scale) as a function of the oscillator length $r$ for the chiral non-relativistic constituent quark model (upper figure) or the MIT bag radius $R$ for the cloudy bag model (lower figure). The dashed lines indicate the spin contribution of the valence quarks when the depolarizing effects of the meson cloud are neglected.

defined in accordance with Eq. (6).

Mesons thus contribute important corrections to our qualitative understanding of the nucleon structure. Here, we have shown, in particular, the effects of the virtual meson cloud on the reduction of the Gottfried sum rule, the appearance of non-zero strange quark matrix elements in the nucleon, and why the valence 
quarks' contribution to the nucleon spin may be smaller than naively anticipated. Therefore, any complete, quantitative evaluation of the nucleon structure should include the effects of the meson cloud.

\section{References}

1. See e.g., G.A. Miller, Intern. Rev. Nucl. Phys. 1 (1984) 189 ; A.W. Thomas, Prog. Nucl. Phys. 13 (1984) 1.

2. P. Amaudruz et al., Phys. Rev. Lett. 66 (1991) 2712.

3. J. Ashman et al., Phys. Lett. B206 (1988) 364; Nucl. Phys. B328 (1989) 1.

4. See e.g., D.B. Kaplan and A.V. Manohar, Nucl. Phys. B310 (1988) 527; R.L. Jaffe and A.V. Manohar, Nucl. Phys B337 (1990) 509; D.B. Kaplan, Phys. Lett. B275 (1992) 137.

5. W-Y.P. Hwang and E.M. Henley, Ann. Phys. (NY) 129 (1980) 47; E.M. Henley, G. Krein and A.G. Williams, Phys. Lett. B281 (1992) 178; T. Frederico et al., Phys. Rev. C46 (1992) 347; E.M. Henley, G. Krein, S.J. Pollock, and A.G. Williams, Phys. Lett. B269 (1991) 31.

6. G.T. Garvey et al., Phys. Lett. B289 (1992) 249; T. Suzuki, Y. Kohyama, and K. Yazaki, Phys. Lett. B252 (1990) 323.

7. D.H. Beck, Phys. Rev. D39 (1989) 3248; R. D. McKeown, Phys. Lett. B219 (1989) 140; E.J. Beise and R.D. McKeown, Comm. Nucl. Part. Phys. 20 (1991) 105.

8. W. Koepf, E.M. Henley and S.J. Pollock, Phys. Lett. B288 (1992) 11; W. Koepf and E.M. Henley in print by Phys. Rev. C; B.R. Holstein, Proc. Parity Violation in Electron Scattering eds. E.J. Beise and R.D. McKeown (World Scientific Singapore, 1990) pp. 27-43.

9. For an analysis and references, see G. Altarelli, P. Nason and G. Ridolfi, Phys. Lett. B320 (1994) 152.

10. K. Gottfried, Phys. Rev. Lett. 18 (1967) 1174.

11. R.D. Field and R.P. Feynman, Phys. Rev. D15 (1977) 2590, and Nucl. Phys. B136 (1978) 1.

12. A.W. Thomas, Phys. Lett. 126B (1983) 97; E.M.Henley and G.A. Miller, Phys. Lett. B251 (1990) 453.

13. J.D. Sullivan, Phys. Rev. D5 (1972) 1732.

14. A.I. Signal and A.W. Thomas, Phys. Lett. B211 (1988) 481, and Phys. Rev. D40 (1989) 2832; A.I. Signal, A.W. Schreiber, and A.W. Thomas, Mod. Phys. Lett. A6 (1991) 271, S. Kumano, Phys. Rev. D43 (1991) 59; S. Forte, Phys. Rev. D47 (1993) 1842; W. Melnitchouk, A.W. Thomas, and A.I. Signal, Z. Phys. A340 (1991) 85; S. Kumano and J.T. Londergan, Phys. Rev. D46 (1992) 457. 
15. P.L. McGaughey et al., Phys. Rev. Lett. 69 (1992) 1726.

16. E.J. Eichten, I. Hinchliffe and C. Quigg, Phys. Rev. D47 (1993) R747; A. Szczurek and J. Speth, Nucl. Phys. A555 (1993) 249.

17. T.P. Cheng and R.F. Dashen, Phys. Rev. Lett. 26 (1971) 594 and Phys. Rev. D13 (1976) 216; T.P. Cheng, Phys. Rev. D13 (1976) 2161; C.A. Dominguez and P. Langacker, Phys. Rev. D24 (1981) 190; J.F. Donoghue and C.R. Nappi, Phys. Lett. 168B (1986) 105; J.F. Donoghue, Ann. Rev. Nucl. Part. Sci. 39 (1989) 1; J. Gasser, H. Leutwyler and M.E. Sainio, Phys. Lett. B253 (1991) 252.

18. H. Genz and G. Höhler, Phys. Lett. 61B (1976) 389; J. Ellis, E. Gabathuler and M. Karliner, Phys. Lett. B217 (1989) 173; B.L. Ioffe and M. Karliner, Phys. Lett. B247 (1990) 387; ASTERIX Collaboration, Phys. Lett. B267 (1991) 299.

19. D.B. Kaplan and A.E. Nelson, Phys. Lett. B175 (1986) 57; ibid. B179 (1986) 409(E); ibid. B192 (1987) 193.

20. L.A. Ahrens et al., Phys. Rev. D35 (1987) 785.

21. B. Adeva et al., Phys. Lett. B302 (1993) 533.

22. P.L. Anthony et al., Phys. Rev. Lett. 71 (1993) 959.

23. F.E. Close and R.G. Roberts, Phys. Lett. B316 (1993) 165.

24. Bates experiment No. 89-06, R.D. McKeown and D.H. Beck spokespersons.

25. CEBAF proposal No. PR-91-004, E.J. Beise spokesperson; CEBAF proposal No. PR-91-010, J.M. Finn and P.A. Souder spokespersons; CEBAF proposal No. PR-91-017, D.H. Beck spokesperson.

26. LAMPF proposal No. 1173, W.C. Louis, spokesperson.

27. G. Karl, Phys. Rev. D45 (1992) 247.

28. K. Steininger and W. Weise, Phys. Rev. D48 (1993) 1433.

29. R.L. Jaffe, Phys. Lett. B229 (1989) 275.

30. T.D. Cohen, H. Forkel and M. Nielsen, Phys. Lett. B316 (1993) 1.

31. N.W. Park, J. Schechter and H. Weigel, Phys. Rev. D43 (1991) 869.

32. M.J. Musolf and M. Burkardt, CEBAF preprint TH-93-01 (1993), in print by Z. Phys. C.

33. S.C. Phatak and S. Sahu, Phys. Lett. B321 (1994) 11.

34. P. Zenczykowski, Phys. Rev. D29 (1984) 577 .

35. Y. Nogami and N. Ohtsuka, Phys. Rev. D26 (1982) 261.

36. J.Ellis and R.L. Jaffe, Phys. Rev. D9 (1974) 1444; ibid. D10 (1974) 1669.

37. J.D. Bjorken, Phys. Rev. 148 (1966) 1467; Phys. Rev. D1 (1970) 1376.

38. J. Ellis and M. Karliner, Phys. Lett. B313 (1993) 131, and CERN preprint TH.7022/93.

39. B. Adeva et al., Phys. Lett. B320 (1994) 400. 